\title{
Elementos para pensar la formación pedagógica y didáctica de los profesores en la universidad ${ }^{1}$
}

\section{Elements to think about the Pedagogical and didactic formation of the professors in the University}

Hamlet Santiago González $^{2}$
Rusby Malagónlez

Citation / Para citar este artículo: González, H. S. E Malagón, R. (2015). Elementos para pensar la formación pedagógica y didáctica de los profesores en la universidad. Colomb. Appl. Linguist. J., 17(2), pp. 290-301.

Received: 12-Dec-2014 / Accepted: 29-May-2015

DOI: http://dx.doi.org/10.14483/udistrital.jour.calj.2015.2.a08

\begin{abstract}
La formación de los profesores universitarios es un tema fundamental a la hora de pensar la calidad de la enseñanza de los nuevos profesionales y cualificar el aporte del recurso humano en la sociedad. El presente artículo de reflexión propone, a partir de la discusión de los análisis realizados por algunos autores en el ámbito nacional e internacional, la realización de un análisis de la formación de los profesores universitarios en el caso colombiano, así como nuevas formas para implementar su desarrollo. Los principales aportes propuestos al debate son: a) Es fundamental el establecimiento de una política unificada de formación de docentes en Colombia, la cual se debe desarrollar incluso en contra de la falta de voluntad política de los entes gubernamentales. b) Se propone la recuperación del saber pedagógico como forma de abordaje para realizar la formación de profesores universitarios, haciendo énfasis en el aspecto comunicacional. c) Finalmente se destaca la importancia que tiene la implementación de procesos de investigación pedagógica y educativa, los cuales son escasos en nuestro medio.
\end{abstract}

Keywords: enseñanza, formación de docentes, pedagogía, saber, técnica didáctica

\section{Resumen}

University Teachers training is a key aspect when regarding the quality of education imparted to future professionals and when qualifying the contribution of human resource to society. Taking into account discussions of analysis suggested by local and foreign authors, this reflection paper proposes an analysis of teachers training at university level in Colombia and paths to contribute on its development. The main contributions to the debate are: a) establishing a unified policy of teachers training in Colombia is essential, despite the lack of political desire from government agencies; b) recovering the relevance of the pedagogical knowledge in order to improve teachers training by emphasizing on communicational aspects; c) finally,

1 Producto del Seminario Formación, Educación, Pedagogía y Didáctica, dirigido por el Profesor Carlos Eduardo Vasco Uribe, en el Doctorado Interinstitucional en Educación (DIE), sede Universidad Distrital Francisco José de Caldas (agosto-diciembre de 2012), como requisito para el cumplimiento de créditos de movilidad dentro del proceso de formación doctoral de los autores, en el Doctorado en Ciencias Sociales Niñez y Juventud, Universidad de Manizales-CINDE. Igualmente presenta desarrollos del Proyecto de Formación Pedagógica y didáctica de los profesores de la Universidad Distrital Francisco José de Caldas del Comité institucional de Currículo en el marco del Plan de desarrollo vigente en dicha institución.

2 Universidad Distrital Francisco José de Caldas, Bogotá, D.C., Colombia. hsgonzalezm@udistrital.edu.co

3 Universidad Pedagógica Nacional, Bogotá, D.C., Colombia. rusbyma@gmail.com 
highlighting the importance of carrying out pedagogical and educational research projects which seldom take place.

Keywords: education, knowledge, pedagogy, teacher education, teaching technique

\section{Introducción}

La pedagogía y la didáctica son fundamentales en los procesos de formación de los profesores universitarios. Estas áreas proveen elementos para una adecuada co-construcción del conocimiento con los estudiantes. La formación de los profesores universitarios es un tema fundamental a la hora de pensar la calidad de la enseñanza de los profesionales y cualificar el aporte del recurso humano en la sociedad. Sin embargo, este asunto ocupa un segundo lugar en muchas instituciones universitarias. Es importante mencionar que por muchos años se ha privilegiado el conocimiento que los profesores tienen sobre su disciplina, independientemente del conocimiento pedagógico y su aplicación en los procesos de enseñanza con los estudiantes. En el presente artículo de reflexión se precisarán algunos conceptos básicos a fin de tener claridad de la magnitud del problema, así como algunas posibilidades de abordaje para pensar y aplicar políticas de formación de profesores.

El itinerario a seguir en el texto inicia con algunas definiciones sobre la formación, la pedagogía y la didáctica, con la intención de fundamentar la forma como se asumirán tales conceptos en el desarrollo de la reflexión. Posteriormente, nos referiremos a algunos modelos de formación que han construido los docentes universitarios, los cuales abordan las formas y modos que aquellos utilizan para reflexionar sobre su labor. A continuación se plantean algunos elementos en relación con la importancia de establecer una política unificada de formación de docentes en el marco de un sistema nacional de formación de profesores en Colombia. Posteriormente, se propone el saber pedagógico como uno de los ejes para realizar los procesos de formación, haciendo énfasis en el aspecto comunicacional. Finalmente, se destaca la importancia de la investigación pedagógica y educativa.

\section{Formación, pedagogía y didáctica}

En este primer apartado se realizará una fundamentación básica de los conceptos de formación, pedagogía y didáctica en aras de avanzar en términos de claridad y tener una base para la reflexión en torno a los modelos de formación de los docentes universitarios.

La formación podría definirse como un proceso constante de inculturación a través del cual los seres humanos se apropian, recogen, adoptan, se construyen en la cultura en la que se encuentran inmersos. Es el resultado de una mediación por medio de la cual los seres humanos se hacen parte de su cultura, asumen sus reglas, sus condiciones, sus normas, en respuesta a las necesidades que en un momento dado les demande. Al respecto Vasco sostiene:

Se trata de formar al nuevo miembro de la familia, el clan o la etnia, en el sentido de 'darle la forma' prevista y valorada por la cultura. Pero desde el punto de vista de ese nuevo ser que crece en la cultura, si lo tomamos como agente principal de su propia formación, su perspectiva se expresa mejor con el verbo reflexivo: se trata de formarse como persona, en el sentido de desarrollar su propia forma interna con todas las potencialidades de su singularidad y su originalidad. (2011b, p. 17).

El concepto de pedagogía ha sido objeto de estudio desde múltiples perspectivas; tradicionalmente el uso de la palabra se ha referido a una de las actividades propias de la profesión docente, pero las nuevas dimensiones y significados que adquiere la reflexión sobre lo que es la formación y la educación, lo sitúan en un contexto que hace difusa y poco precisa su conceptualización; el conocimiento pedagógico puede entenderse como esas grandes reflexiones sobre los procesos formativos y educativos. En palabras de Vasco: 
Las reflexiones, saberes y discursos más o menos formales o disciplinados sobre la formación y la educación conforman la pedagogía. En esta propuesta de restricciones discursivas que espero faciliten el diálogo y la comunicación, la pedagogía no es necesariamente una filosofía, ni una ciencia, ni una disciplina académica, aunque puede serlo para algunas personas y en algunas instituciones: más bien, para mí, la pedagogía es ese acumulado de reflexiones, saberes y discursos sobre la formación y la educación. (2011b, p.21)

La didáctica podría definirse como el campo del conocimiento donde se precisa, estudia y analiza los métodos y técnicas que favorecen los procesos de enseñanza; es la parte de la pedagogía donde se reflexiona y se recrean caminos o situaciones para que los estudiantes alcancen una comprensión sobre una temática en particular, "así, la didáctica de un área o de una asignatura escolar como disciplina académica centrada en la enseñanza de saberes específicos sería parte de la pedagogía y no podría oponerse a esta". (2011b, p. 21). En otra parte, el mismo autor señala: "La didáctica cubre la reflexión sobre todos los aspectos de las relaciones del maestro con sus alumnos y sus micro entornos desde el punto de vista de la enseñanza" (Vasco, 1990, p.113).

Los conocimientos pedagógicos y didácticos deberían estar relacionados de forma directa con la labor de los profesores universitarios; pero cuando se revisa la literatura académica en esta temática, se hace evidente la fortaleza de esos conocimientos a nivel escolar, lo cual se muestra en la gran cantidad de estudios e investigaciones, en contraposición con la fragilidad en los contextos universitarios, dada la insuficiencia de los trabajos que retoman esta problemática.

Los profesores han sido objeto de múltiples miradas en la historia, en su gran mayoría, de invisibilización y subordinación, pero no se puede negar que diferentes movimientos sociales, políticos y las reflexiones suscitadas por equipos de intelectuales liderados por docentes, han alcanzado construcciones teóricas que han posicionado la profesión.

A partir de las precisiones esbozadas anteriormente, se podría afirmar que cuando nos referimos a la formación pedagógica y didáctica de los profesores universitarios estamos dando cuenta de aquel proceso de inculturación y apropiación de las reflexiones, saberes, discursos y modos de actuar que favorecen que los profesores reconozcan y dimensionen la importancia de los factores involucrados en el desarrollo social, emocional y cognitivo de los futuros profesionales; conocimiento que deben tener los profesores de cualquier nivel educativo. Al respecto Hernández afirma que:

Se requiere entonces, docentes investigadores
capaces y preparados que no solamente
conozcan y manejen el contenido científico
de su campo disciplinar, sino que además,
tengan la formación y capacitación para
escudriñar los conflictos, necesidades y
problemas del hombre y de la sociedad, que
tengan las competencias necesarias, en el
manejo del discurso pedagógico-investigativo
para que guíen y orienten a sus estudiantes
a determinar los puntos álgidos, sobre los
que debe intervenir para lograr los cambios y
transformaciones esperadas. (2007, p.1)

Modelos que han construido los docentes universitarios en relación con la formación pedagógica y didáctica

Desde nuestra propia experiencia como profesores universitarios, observamos cómo algunas iniciativas de formación referidas a aspectos relacionados con la formación pedagógica y didáctica, no presentan tanto interés. Según Vasco (2001), lo anterior se debe principalmente a tres aspectos:

Inicialmente el aspecto sociológico, que se encuentra estrechamente relacionado con las instituciones y sus dinámicas, pues existen, según el autor en mención, imaginarios que desvirtúan este tipo de saberes, se privilegia el trabajo investigativo sobre la labor docente, se premian las publicaciones relacionadas con temáticas disciplinares y se 
menosprecian las que se hallan relacionadas con saberes pedagógicos y didácticos; se promueven actitudes que descalifican la formación de postgrados que favorezca la cualificación del docente en su actuar pedagógico. De esta manera, al decir de Vasco:

Todos los mensajes respecto a la minusvaloración de la docencia universitaria son claros y coherentes, así no sean explícitos; con frecuencia son directamente contradictorios con las frases grandilocuentes de las misiones y visiones de las universidades, y con los discursos de rectores y decanos el día del profesor. (2001, p.5)

En esta línea de pensamiento, en el rol de los docentes universitarios existen concepciones sociales sobre la labor docente y su condición social que desvirtúan y descalifican los conocimientos pedagógicos y didácticos. Aunque no se hagan explícitos ni se verbalicen discursos contrarios, en la práctica real de las universidades es notorio el menosprecio que se siente en relación con este saber, al igual que frente a la representación social de la profesión docente. Al respecto Imbernon señala la importancia de superar el viejo supuesto de que el profesor universitario es aquél que solo conoce la materia científica, pues, aunque lógicamente el profesorado universitario se forma en el contenido científico de la materia que enseña e investiga, quizá sea muy escasa, o nula, la actitud del profesorado respecto a la formación en cómo se ha de transmitir y compartir ese conocimiento con su alumnado (2011, p.388).

De igual forma, Giusti (2007) reflexiona acerca de la importancia de la formación pedagógica para el profesor universitario, teniendo en cuenta cuáles han sido las razones por las cuales la formación pedagógica del docente universitario y la tarea de enseñar en la universidad ha sido relegada a segundo plano a lo largo de la historia de la educación superior. Concluye luego de un análisis histórico, que hay factores que influyentes en el proceso. Por ejemplo, la docencia universitaria ha sido una actividad de menor importancia, como lo expresa a continuación:
Se creía que el entrenamiento profesional podría ser ofrecido por cualquier persona que supiese realizar bien determinado oficio. O sea, se creía, como muchos aún hoy creen, que 'quien sabe hacer, sabe automáticamente enseñar', no habiendo mayores preocupaciones con la necesidad de una preparación pedagógica de los docentes [...]. (2007, p.120)

Pero no solo la universidad contribuye a la desvalorización de la enseñanza ahondando en el aspecto legal de la educación superior, desde el estudio realizado en Brasil, Giusti evidencia que no existe un amparo legal para el estímulo de la formación pedagógica delos docentes universitarios ${ }^{4}$ lo que "[...] refleja y favorece la creencia de que la formación específica para la docencia universitaria no es necesaria" (2007, p.120). Las universidades imparten la formación que mejor consideran a sus estudiantes de pregrado y posgrado, que en ocasiones "[se] aleja de cualquier discusión sobre temáticas pedagógicas, y no toma en cuenta, incluso, que los elementos clave del proceso de investigación (sujetos, tiempo, conocimiento, resultados y métodos) no son semejantes a aquellos necesarios a la actividad de enseñanza" (2007, p.121). Las tareas esperadas actualmente del docente universitario no se resumen a enseñar e investigar, sino que muchas otras hacen parte de su trabajo (evaluar, publicar, organizar eventos, gestionar procesos académicos dentro y fuera de la institución, entre otras), que se quedan por fuera de los programas de formación. "Todos estos aspectos nos llevan a concluir que la formación de los docentes universitarios requiere de ir más allá de los límites del conocimiento de determinada disciplina y de la adquisición de habilidades para la realización de investigación" (2007, p.122).

Giusti enfatiza la importancia de la preparación pedagógica y didáctica en forma sistemática, señalando que la formación docente debe realizarse de forma continua, en la medida en que

4 Como se verá en otras investigaciones aquí reseñadas Zea et al., 2005; Navarro, 2009), esta situación no solo sucede en Brasil, sino también en otros países latinoamericanos. 
Comprendemos que la formación de los docentes universitarios no se acaba en su preparación inicial, realizada en especial en los cursos de posgrado, siendo que el proceso de formación empieza antes del inicio de la carrera profesional, cuando el futuro profesor es apenas un estudiante. En ese momento, recibe sus primeros ejemplos de conducta docente. De ahí que el proceso formativo continúa a lo largo de toda la carrera del profesor, constituyéndose en un proceso de perfeccionamiento constante. (2007, p.122)

Igualmente, resalta la importancia del trabajo reflexivo en la formación de profesores, a través de la implementación de una formación pedagógica; sin embargo esta no debe limitarse a los aspectos prácticos del quehacer docente, sino que debe englobar también cuestiones relativas a lo ético, lo afectivo y lo político. Asimismo, plantea que el docente debe estar siempre en proceso de formación y auto evaluación.

El segundo aspecto mencionado por Vasco (2001) es el cultural, asociado a una cierta pérdida de estatus que el profesor de escuela ha tenido y de cómo esta situación llevó a los docentes universitarios a relacionar la pedagogía y la didáctica con una profesión devaluada; esta razón los motivó a tomar distancia entre su labor y dichos temas. También en algunas ocasiones se conserva la idea de que a mayor cantidad de alumnos, mayor es el status del profesor. Como lo enuncia Vasco (2001), a partir de sus observaciones y diálogos como en su labor de formador de educadores y científicos por más de tres décadas en Colombia

Esta actitud persiste aún en muchos departamentos de matemáticas, estadísticas, física, química y en grado menor en biología, pero muchos profesores de otras disciplinas añoran el poder de sus colegas de las ciencias naturales y las matemáticas y tratan de imitar su prepotencia. (2001, p.6)

El tercero y último aspecto, que puede ayudar a develar las razones por las cuales el conocimiento pedagógico y didáctico ha sido menospreciado en los contextos universitarios, se encuentra relacionado con la historia; según Vasco (2001), la incursión de la tecnología educativa y, con ella, el diseño de programas curriculares al estilo instruccional de corte conductista, que introducen una idea de la didáctica como el seguimiento de recetas y modos de actuar, ocasionó que los docentes universitarios se distanciaran y dudaran de este conocimiento.

Los elementos que a grosso modo se han enunciado sobre la valoración que hacen los profesores universitarios acerca del conocimiento pedagógico y didáctico, pone en relieve las razones (válidas, infundadas, malinterpretadas $\mathrm{o}$ distorsionadas) que han ocasionado que estos profesores busquen ser reconocidos como profesionales de otra condición, con otras competencias y con otras responsabilidades sociales.

\section{Formación pedagógica y didáctica de los profesores universitarios en Colombia}

Las acciones que se han adelantado frente a la formación de profesores universitarios, se circunscriben a unos cuantos documentos referidos más a las políticas de calidad de las instituciones y al desarrollo de programas específicos en diferentes escenarios universitarios, mas no a emprender reflexiones con el ánimo de constituir un campo de investigación sobre las problemáticas sociopolíticas, culturales y cognitivas involucradas de la formación de los futuros profesionales.

En últimas, estas reflexiones deberían estar asociadas con preguntas como: ¿Qué clase de ciudadanos se quieren formar? ¿Qué clase de profesionales necesita un país como Colombia? ¿Cómo lograr que los futuros profesionales se asuman como partícipes del crecimiento del país? Para Giraldo, Abad y Díaz:

Se debe pensar en tener instituciones de
educación superior que desde su misma
estructura académica adelanten reformas
curriculares que fortalezcan los núcleos
básicos de la formación en disciplinas y
profesiones y reduzcan los contenidos


meramente informativos; y que tengan la capacidad para responder adecuadamente a las demandadas de la sociedad, como también para plantearle nuevas alternativas y posibilidades de desarrollo. (2002, p.14).

Evidencia de la poca importancia que reviste la formación de profesores universitarios en el país son las escasas investigaciones realizadas y la forma aislada y desarticulada como se encuentra la información, lo cual permite inferir que esta categoría de conocimiento merece ser explorada y constituida en un campo de estudio.

Parra, Ecima, Gómez y Almenares (2010) realizaron una investigación que versó sobre las estructuras, métodos, estrategiasyprocedimientos de las rutas de formación de los profesores universitarios en las universidades privadas colombianas, con el propósito de conocer cómo están formando las universidades privadas a sus profesores y proponer criterios y lineamientos generales para el diseño de rutas de formación docente. En dicha indagación encontraron que todos los directivos docentes entrevistados manifestaron que entre las mayores dificultades de sus docentes estaba la carencia de competencias pedagógicas para favorecer procesos de aprendizaje, que las estrategias utilizadas por los docentes estaban estrechamente relacionadas con las actitudes de los estudiantes, con la deserción estudiantil y con los resultados de los ECAES. Los directivos docentes entrevistados en el estudio también afirmaron que las necesidades de formación de profesores estaban asociadas al aprendizaje de una segunda lengua, al uso de las TIC, al desarrollo de habilidades investigativas asociadas a su disciplina y a la vinculación de las temáticas que se abordan en el aula con la vida de los estudiantes. En este estudio, las tendencias de los programas de formación de profesores muestran un énfasis en el desarrollo de diplomados y especializaciones creadas por las universidades para sus propios docentes. Son muy pocos los casos de cofinanciaciones para maestrías y doctorados relacionados con la formación pedagógica.

El panorama de las universidades privadas ha iniciado importantes acciones en términos de la formación de sus docentes, especialmente en lo que respecta a la formación pedagógica, pero es importante seguir trabajando en esta línea. Al respecto Hernández plantea:

Las universidades del sector privado en su estatuto docente registran esta prioridad, pero no se implementa. Tienen cursos de capacitación esporádicos para inducción de docentes, ignorando que la práctica pedagógica como opción de vida se constituye en un requisito básico para elevar la calidad del servicio educativo que prestan, entendida la educación como 'formación'. (2007, p.6)

Tal situación ha cambiado en los últimos años, dada la importancia que ha venido adquiriendo en la educación universitaria los aspectos pedagógicos en la formación. Al respecto Cortés y González (2006), afirman que la formación del docente universitario debe tener en cuenta como objetivos: la estimulación del mejoramiento continuo de la calidad, la eficacia y la eficiencia de la docencia y la investigación; promover la preparación y formación de personal docente más eficiente en el campo de la docencia y la investigación; promover el perfeccionamiento integral del profesorado de escalafón para incrementar su formación, promover la formación de personal especializado, sobre todo en lo referente a nuevos enfoques, técnicas y procedimientos de enseñanza, aprendizaje y evaluación; cooperar con cursos, programas de estudios de postgrado a nivel de especialización, maestría y doctorado; promover el perfeccionamiento del personal docente y administrativo; detectar las necesidades de adiestramiento, capacitación y perfeccionamiento docente; fomentar y desarrollar investigaciones, trabajos experimentales y de innovación educativa en las universidades.

De igual manera, es importante resaltar los planes de cualificación docente de las universidades en las que existen programas de formación, que se encuentran estructurados de acuerdo con las tendencias internacionales de la educación, la evaluación de calidad, los procesos de acreditación y la necesidad de retener a los estudiantes. Dichos campos, demandan que las universidades pongan todo su empeño en responder a las nuevas demandas. Para Gartner: 
Los vertiginosos cambios económicos y sociopolíticos de las últimas décadas han conducido a las universidades, en particular las universidades públicas, a una revolución académica en la que no solo se transforman las actividades de generación, transmisión y utilización del conocimiento, sino también sus formas de gestión en el marco de dinámicas sociales que, en muchos aspectos, se han sobrepuesto a lo que ellas han querido ser y a las rutas que han deseado recorrer. Lo cierto es que están sobre el tapete su reorientación externa y su reconfiguración misional y de gestión interna. Contexto en el cual empiezan a consolidarse exigencias de calidad que aún enfrentan muchas dificultades de carácter técnico, conceptual y político. (2012, p.1).

Investigadores de universidades privadas $\mathrm{y}$ públicas (Tamayo, 2007; Parra, C; Ecima, I; Gómez, M. E Almenares, F. 2010; Arias, 2014; González, 2013) en el país manifiestan la necesidad de la formación pedagógica y didáctica de los profesores universitarios, con argumentos de diferente orden: los requerimientos y exigencias que plantea el mundo globalizado; las nuevas formas de acceder a la información; los procesos de acreditación; el discurso de la calidad; las competencias; el desarrollo económico; la relación entre la labor del profesor universitario y el desarrollo de habilidades investigativas en los estudiantes; la enseñanza de campos específicos del conocimiento; el saber pedagógico y la ética del profesor universitario, etc. Estos documentos se constituyen en elementos importantes para la reflexión sobre la formación de profesores universitarios, pero no afectan ni determinan las políticas administrativas y académicas que favorecen la materialización de proyectos que transformen la realidad de las instituciones.

Es claro que la formación pedagógica en los programas de pregrado es un campo un tanto descuidado en las universidades, desde el supuesto de que el conocimiento sobre la disciplina particular (química, biología, etc.) suple la necesidad de formación en pedagogía y didáctica; cuando la institución quiere aportar a la formación de los profesores, lo hace a través de especializaciones o diplomados que abordan temáticas sobre el conocimiento pedagógico general, desligado del conocimiento asociado a la enseñanza de la disciplinas particulares. Esto hace explícita la tensión y divergencias que históricamente se han planteado en algunas facultades de ciencias y educación, en relación con el conocimiento pedagógico y el conocimiento disciplinar.

\section{La importancia de establecer una política unificada de formación docente en el país}

La formación de docentes no puede tratarse de manera aislada. De hecho, lo ideal es tener un sistema general de formación que agrupe todos los niveles educativos de forma articulada. Al respecto, Vasco (2011a) en su artículo "¿Es posible un sistema nacional de formación de docentes?" presenta algunas ideas emanadas del trabajo que realizó en compañía de Alberto Martínez Boom y Eloísa Vasco, en un estudio realizado por el MEN sobre la viabilidad de una política de formación de docentes en Colombia. Dicho diagnóstico no fue muy alentador, en la medida en que a nivel institucionalgubernamental no están dadas las condiciones necesarias para establecer una voluntad política al respecto. En este documento se sitúan, entre otros, los siguientes aspectos:

- La discusión sobre el ciudadano que queremos formar y sobre el país que queremos tener. Asunto capital a la hora de definir el derrotero a seguir en materia educativa.

- Interrogantes de capital importancia tales como: ¿Quién formará a los formadores de formadores? En ese sentido caben las preguntas: ¿Acaso los funcionarios de escritorio, carentes de experiencia educativa, que a partir de la enunciación de políticas imponen sin discusión sus dictámenes? ¿Los mismos maestros a partir de su propio saber pedagógico? ¿Los expertos e investigadores? o ¿̇al vez los mismos docentes deben plantear su aporte a la formulación de dichos procesos?

- ¿El maestro nace, o se hace? En este sentido, "Si el maestro nace y no se hace, si la pedagogía es un arte solo comparable con el de los pocos pintores consumados o compositores inspirados, no vale la pena diseñar ningún 
sistema de formación de docentes" (Vasco, 2011a, p.26).

- ¿El desmedro por la educación será falta de visión, descuido o sencillamente una estrategia intencional para mantener las castas en el poder con los subalternos necesarios para perpetuar sus intereses de dominación?

- Cambiar primero el sistema para luego cambiar la escuela dando lugar a la posición anti-escuela. Esta fue ratificada gracias a una interpretación de Foucault, que plantea la escuela como cárcel. Es importante analizar la viabilidad de dicha propuesta o distinguir si es una posición demasiado radical.

- La evaluación de docentes, suscitada a partir de la intervención de la banca multilateral, sus compromisos adquiridos y en la estabilidad que detentan los profesores del antiguo régimen magisterial. El magisterio, desde su dirección sindical, ha sido reacio a la aplicación de la evaluación por competencias. Una posibilidad de vertebrar este asunto de una forma diferente, tendría que ver con el planteamiento de la evaluación en términos de las capacidades que tienen los docentes para realizar su labor de enseñanza y no con la forma de evaluaciónsanción como tratamiento que se le quiere dar tradicionalmente desde las instancias de poder.

Sin embargo, a pesar de los problemas estructurales planteados anteriormente, hoy se ha retomado el tema y la nueva administración del Ministerio de Educación Nacional ha concertado una agenda en la cual hay activa participación de la Asociación Nacional de Escuelas Normales Superiores ASONEN, Secretarías de Educación y la Asociación Colombiana de Facultades de Educación (ASCOFADE) (Colombia aprende, 2012).

Aquí llama la atención la gran velocidad con la cual avanza el proceso, a pesar de existir supuestas posiciones irreconciliables y fuertes dificultades entre los sindicatos, las facultades de educación y los lineamientos institucionales del Ministerio de Educación Nacional. De hecho, no sería la primera vez que, tras el planteamiento de dichos ejercicios democráticos, se ejecutan acuerdos políticos preestablecidos de antemano, simulando un trabajo mancomunado que en realidad solo es una forma de legitimar lo que ya se ha establecido previamente.

Finalmente es importante señalar que, en la enunciación de una política de formación de docentes, es fundamental encontrar un nexo claro entre la formación inicial de pregrado y la formación continuada de los docentes en ejercicio, de tal manera que exista una complementariedad entre tales niveles.

\section{El saber pedagógico como referente en la formación de profesores}

Las acciones iniciales de formación deben plantear una dinámica de formación que reconozca el saber propio del profesor universitario y minimice procesos de formación ajenos a su historia. Según Hernández:

El docente, inmerso en el mundo universitario, debe orientar su acción hacia la construcción de su propio saber pedagógico, competencia que le permite proyectar desde su rol una nueva universidad [...] además, desarrollar creativas habilidades, que fueran útiles para las demandas y exigencias de la sociedad a nivel regional, nacional y mundial. $(2007$, p. 6)

En este sentido, es necesario propiciar espacios para que los profesores universitarios compartan y reflexionen sobre su saber pedagógico; tal estrategia reivindica que la actividad de formación pedagógica del profesor se debe realizar en su cotidianidad. Se podría contar con la presencia de algunos invitados que acompañen el trabajo y muestren experiencias significativas al respecto. Desde esta perspectiva, entrarían a ser el centro de la formación de los profesores universitarios, sino que aportarían en el ejercicio metacognitivo y en la visibilización de otras formas de leer la realidad. Al respecto Velásquez afirma que:

La comunidad académica en las instituciones de educación superior necesita revalorar su trabajo pedagógico y su práctica docente, situarse en meta objetivos que vayan más allá de los instruccionales de un curso y centrarse 
en el aprendizaje, descentrar su acción de la enseñanza propiamente dicha y de la práctica aún muy generalizada, que encuentra su expresión fundamental en la cátedra magistral, fundamentalmente expositiva. (2007, p. 3)

El giro en la forma de asumir la formación haciendo énfasis en el aprendizaje de los estudiantes, ha generado una profunda transformación en los procesos de formación que se llevan a cabo en la Universidad. Por esta vía Zabalza, plantea:

Quien aprende no es un grupo sino cada sujeto y cada uno de ellos a su manera (quod recipitum recipitur in modo recipientis). Una docencia basada en el aprendizaje de nuestros estudiantes nos obliga a estar pendientes de cada uno de ellos, a supervisar el proceso que va siguiendo, a facilitar su progreso a través de los dispositivos didácticos cuyo dominio se nos supone como profesionales de la enseñanza. Por eso, enseñar (en el sentido de explicar) es bien distinto de 'facilitar el aprendizaje'. Para lo primero pudiera ser suficiente (aunque no siempre) saber mucho del tema que explico, para lo segundo hay que saber, además, de otras cosas (cómo motivar, cómo organizar procesos de aprendizaje adaptados a los estudiantes, cómo supervisar y tutorizar sus actividades, cómo evaluarlos y ayudarles a resolver las dificultades, etc.). (2014, p.13)

\section{La importancia de la comunicación en la formación docente}

La concreción de lo pedagógico se realiza a partir de un acto comunicativo, lo cual involucra los diferentes factores que intervienen en la comunicación. Los profesores universitarios generalmente reducen el problema de la enseñanza a emitir un mensaje claro y con ello creen suplir las necesidades pedagógicas, sin tener en cuenta que los significados que le asocia el estudiante al mismo, dependen de los contextos específicos de cada estudiante, así como de los significados que se le otorgan a la información emitida, dependiendo de su experiencia particular y de los significados que a través de la historia se le han dado al mismo mensaje. En este sentido, Peña afirma:
La importancia que tiene el lenguaje en la formación universitaria puede sintetizarse en sus tres funciones principales: una función comunicativa, en cuanto sirve como instrumento para enseñar, evaluar y hacer público el conocimiento; una función social, como mediador en las relaciones interpersonales, los acuerdos y los proyectos cooperativos; y una función epistémica, como herramienta intelectual y de aprendizaje. EI énfasis que se la ha dado a la primera de estas funciones ha hecho que se subestime la importancia que tiene el lenguaje como herramienta psicológica y cultural y como mediación en los procesos de formación propios de la educación superior. (2008, p.1)

La función epistémica y social son fundamentales, en cuanto generan las dinámicas en las que se debaten los diferentes dispositivos de la cultura, desde la interacción social y las formas como circulan en la academia.

La formación de los profesores universitarios pensada desde la comunicación causaría menos resistencias y sería motivante, siempre y cuando se orientara hacia la construcción de pensamiento en relación con los procesos académico-pedagógicos llevados a cabo por los mismos profesores. Aquí, el tema de las TIC aplicadas a nuevas estrategias de aprendizaje potenciado a través de recursos interactivos, sería un aspecto a trabajar a pesar de las resistencias que se plantean al respecto. La utilización de dichas mediaciones debe aprovechar toda la riqueza de las herramientas, a fin de no generar una sub utilización de las mismas o un activismo que finalmente no aporte al objetivo educativo.

De otro lado, es fundamental crear conciencia en los docentes sobre el lenguaje como eje fundamental en la enseñanza. Frente a la recurrencia en la utilización de un lenguaje especializado por parte de los profesores, debe existir un interés por generar la comprensión de las temáticas que se abordan en cuanto a la claridad expositiva de los temas impartidos, pero a la vez una apertura hacia el aprendizaje de nuevos términos, teorías y en general nuevas formas de 
explicar y describir el mundo desde los rigores de la academia.

Al respecto, Zabalza (2006) se refiere a la necesidad de plantear informaciones y explicaciones comprensibles y bien organizadas. Tal gestión de la información supone: una producción comunicativa, que convierte los contenidos en mensajes didácticos, lo cual ocurre al dar una clase o al preparar un material didáctico (p.82). En relación con el mensaje didáctico precisa:

\begin{abstract}
Nuestros mensajes didácticos pueden resultar deficitarios cuando cualquiera de los pasos señalados se produce en condiciones deficitarias, por ejemplo, cuando no tenemos clara la idea que deseamos transmitir, no sabemos de ese tema o lo tenemos poco estudiado. También puede que tengan clara la idea que deseamos explicar pero que tengamos algunos problemas a la hora de disponer de códigos efectivos para codificarla (que nos falte vocabulario, que no seamos capaces de hacer unos buenos gráficos 0 que nos falten los signos necesarios para representarla). (Zabalza 2006, p.83)
\end{abstract}

Sin embargo, esta no es la única dificultad que puede existir en cuanto a la efectividad de lograr el aprendizaje y la comprensión de los estudiantes, frente al conocimiento que expresa el profesor. Asuntos relacionados con la dicción, la velocidad en la transmisión de las ideas y las subjetividades de los alumnos, las condiciones y logísticas del salón de clases auditorio o lugar donde se realiza la actividad educativa, juegan un papel importante en el proceso comunicativo.

Como alternativas para reforzar la comprensibilidad, Zabalsa (2006) plantea el uso de la redundancia de conceptos e ideas al realizar la exposición, cuidando que el recurso no se convierta en contraproducente (p. 85); así como el reacondicionamiento de los propios mensajes, teniendo en cuenta los siguientes elementos: Simplicidad, orden, brevedad-pregnancia ( en cuanto a la expresión lingüística de las palabras necesarias para dar cuenta de las ideas) y la estimulación suplementaria, como forma de llamar la atención de los estudiantes (p. 87). Finalmente, se refiere a la connotación afectiva de los mensajes, que trasmiten la pasión por las temáticas de estudio (p.91). Al respecto son importantes los aportes de Montoya (2014), en referencia a los resultados de su investigación doctoral, en la cual se refiere al discurso epidíctico que efectúan los docentes universitarios en el aula de clase, el cual potencia la orientación, motivación y el compromiso (p. 258). Igualmente, afirma "[...] el epidíctico es una posibilidad poderosa para tejer comunidad, alcanzar consensos inmediatos, la toma de decisiones y poner en circulación en el aula las emociones y también las razones" (Montoya, 2014, p.258).

\section{La investigación pedagógica y educativa}

Este eje es fundamental, dada la misión de la universidad como productora de nuevo conocimiento y en general las necesidades sociales actuales que demandan intervenciones a sus problemáticas concretas. El trabajo investigativo a emprender se puede realizar desde diferentes perspectivas epistemológico-metodológicas, a saber: socio-críticas (investigación-acción pedagógica y educativa); hermenéuticocomprensivas (etnográficas, sistematización, narrativas); arqueológico-genealógicas, ya que dichas perspectivas permiten la construcción de significaciones y sentidos en relación con los fenómenos educativos y pedagógicos a abordar. Cualquiera que sea el fundamento epistemológico, es muy importante documentar y hacer memoria de lo que ocurre en los espacios de docencia en la Universidad, así como iniciar una sistematización de las actividades de extensión y proyección a la comunidad, de tal manera que estas sean fortalecidas y retomadas en las acciones pedagógicas que ejercen los docentes.

\section{Consideraciones finales}

El diseño y ejecución de políticas educativas en materia de formación de docentes, debe integrar todos los niveles de la enseñanza. Ahora bien, a nivel de centros universitarios, se hace importante reivindicar 
el saber pedagógico de los profesores universitarios enfatizando en los aspectos comunicacionales y en la investigación acción pedagógica y educativa, como pilares fundamentales para la elaboración de una estrategia formativa de los profesionales de la educación.

Las anteriores formas de abordaje son fundamentales, en tanto retoman los problemas del desarrollo humano, la transformación sociocultural y la democratización del acceso al conocimiento por parte de los estudiantes. Igualmente, constituyen una invitación a pensar de una manera diferente, en las formas como se concibe el quehacer docente, en términos de la cualificación de los procesos de enseñanza que imparten a sus estudiantes. En este sentido, el presente trabajo reflexiona sobre las implicaciones de los conceptos fundamentales de la formación pedagógica y didáctica, desde sus enunciaciones generales problematiza los modelos que se han planteado y propone nuevas formas para su comprensión y aplicación.

Con el propósito de superar los riesgos que trae consigo la enunciación de una política de formación de profesores unilateral, a priori, vertical, descontextualizada, eminentemente teórica e ideal y que además no consulte las verdaderas necesidades de formación de los profesores, o más aún, que resulte incongruente con la misión y visión de las instituciones universitarias, se plantea una forma de abordaje a partir de la indagación por las formas como efectivamente se concibe y se realiza la formación pedagógica y didáctica de los profesores.

Tal proceso implica el diseño y desarrollo de proyectos de investigación de carácter institucional, que den cuenta de las diferentes dinámicas como se concibe y se realiza dicha formación, a fin de que los resultados de estas indagaciones sean retomadas para la cualificación y aplicación adecuada de una manera general, pero que a la vez tenga en cuenta las diferencias y particularidades propias de cada contexto específico, tanto de las universidades como de las características diferenciales definidas a su interior en las diferentes facultades y programas académicos.
En ese sentido, es importante trazar un trabajo que recoja las diferentes formas de práctica docente que se realizan al interior de la universidad a partir de sus propios protagonistas: los profesores universitarios. Estas formas deben articularse a la enunciación de unos principios y lineamientos, como línea de base e insumo, que provea los elementos necesarios en la construcción de una política de formación desde las dinámicas originales y genuinas en cada institución, a partir de los planteamientos y prácticas cotidianas de los profesores. De esa manera, se superaría la asistematicidad y dispersión como usualmente se viene asumiendo dicha formación.

De igual forma, es fundamental la construcción de programas académicos de postgrado, orientados a la investigación de las dinámicas propias de las instituciones de educación superior que se enlacen al establecimiento de los procesos de formación continuada en los espacios de educación universitaria.

\section{Referencias}

Arias, F. (2014). ¿Des-aciertos en la formación pedagógica y didáctica de los profesores docentes universitarios en Colombia? Bogotá D.C.: Editorial UD. En prensa.

Colombia aprende (2012). Consolidación de la política de Formación de Educadores. Autor. Recuperado de http:// www.colombiaaprende.edu.co/html/productos/1685/w3article-312310.html

Cortés, A. \& González, R. (2006). Programas de formación permanente del Docente Universitario en Venezuela. Omnia, vol. 12, núm. 1, 130-146.

Gartner, L. (2012). Razones para apostarle a la calidad en la educación superior. Recuperado de http://www.cna. gov.co/1741/articles-186502_Razones_Calidad.pdf

Giraldo, U., Abad, D. E Díaz, E. (2002). Bases para una política de calidaden educación superior en Colombia. Recuperado de http://cms-static.colombiaaprende.edu. co/cache/binaries/articles-186502_doc_academico10. pdf?binary_rand $=8818$

González, H. (2013). El Saber Pedagógico de los docentes universitarios. Revista Virtual Universidad Católica del Norte, 39, 95-109. Recuperado de http://revistavirtual. ucn.edu.co/index.php/RevistaUCN/article/view/429/878 
Giusti, G. (2007, julio-septiembre). Formación pedagógica de profesores universitarios: conclusiones de una experiencia brasileña. Educación superior, 36(3) núm.143, 119-132. Recuperado de http://www. redalyc.org/pdf/604/60414308.pdf

Hernández, R. (2007). El saber pedagógico y el docente universitario como investigador. Revistamemorias. com,5(9), Recuperadodehttp://www.revistamemorias. com/articulos9/saberpedagogicol-2007.pdf

Imbernón, F. (2011). La formación pedagógica del docente universitario. Educação. Revista do Centro de Educação, 36(3), 387-396.

Montoya, M. (2014). La fuerza del discurso epidíctico para motivar y tejer comunidad: el caso de profesores y estudiantes de primer semestre de la Universidad Distrital. Colombian Applied Linguistics Journal, 16(2), Recuperado de http://revistas.udistrital.edu.co/ ojs/index.php/calj/article/view/7201/9511

Navarro, T. (2009). La formación del docente universitario en Latinoamérica. Revista Odontológica Mexicana, 1(2), 70-72.

Parra, C., Ecima, I., Gómez, M. \& Almenares, F. (2010, septiembre-diciembre). La formación de los profesores universitarios: una asignatura pendiente de la universidad colombiana. Revista Educación y Educadores, 13(3), 421-452. Recuperado de http:// educacionyeducadores.unisabana.edu.co/index.php/ eye/article/view/1733/2275

Peña, L. (2008). La competencia oral y escrita en la educación superior. Recuperado de http://www. mineducacion.gov.co/1621/articles-189357_archivo_ pdf_comunicacion.pdf

Tamayo, L. (2007, enero-junio). Tendencias de la pedagogía en Colombia. Recuperado de http:// latinoamericana.ucaldas.edu.co/downloads/ Latinoamericana3-1_5.pdf
Vasco, C. (1990). Algunas reflexiones sobre la pedagogía y la didáctica. En: M. Díaz y J. Muñoz (Eds.). Pedagogía, discurso y poder (pp. 107-122). Bogotá: Corprodic.

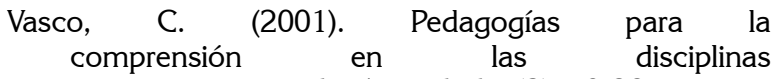
académicas. Uni-Pluri/versidad, 1(3), 19-28.

Vasco, C. (2011a). ¿Es posible un sistema nacional de formación de docentes? Reflexión e Investigación, Revista Editorial del Congreso por una Educación de Calidad, 4, 24-34. Recuperado de http:// porunaeducaciondecalidad.org/fondo-editorial.html

Vasco, C. (2011b). Formación y educación, pedagogía y currículo. En Red Colombiana de Pedagogía (2011). La pedagogía Colombiana. Tomo 1 (pp. 15-35). Cali: REDIPE.

Velásquez, L. (2007). Las redes de investigación virtuales: propuesta de fomento y desarrollo de la cultura investigativa en las instituciones de educación superior. Revista de Universidad y Sociedad del Conocimiento, (RUSC), 4(2), 1-11. Recuperado de http://www.uoc.edu/rusc/4/2/dt/esp/velasquez.pdf

Zabalza, M. (2006). Competencias docentes del profesorado universitario. Calidad y desarrollo profesional. Madrid: Narcea.

Zabalza, M. (2014). Ser (buen) profesor/a en la universidad actual. Ponencia presentada en el I Simposio de formación pedagógica y didáctica para profesores universitarios. Bogotá D.C.: Editorial UD. En prensa.

Zea, C., Atuesta, M., Villegas, G., Toro, P., Nicholls, B. E Foronda, N. (2005). Hacia un modelo de formación continuada de docentes de educación superior en el uso pedagógico de las tecnologías de información y comunicación. Revista Cuadernos de Investigación Universidad EAFIT, 35, 1-64. 